\title{
Cytotoxicity of Nutritional Supplement Galaxy® against HepG2 Hepatocellular Carcinoma
}

David B Jeffs, Alexander C Martin, Erick R Rosenvall, Tyson Fillmore, Katelyn E Barrett and Gary M Booth ${ }^{*}$

Brigham Young University, Department of Plant and Wildlife Sciences, Provo, Utah 84602, USA

*Corresponding author: Gary M Booth, Professor of Plant and Wildlife Sciences, Brigham Young University, 5113 Life Science Building, Provo, Utah 84602, USA, Tel: 801-636-6363; E-mail: gary_booth@byu.edu

Rec date: Jun 18, 2014; Acc date: Nov 7, 2014; Pub date: Nov 9, 2014

Copyright: ( 2014 Jeffs DB, et al. This is an open-access article distributed under the terms of the Creative Commons Attribution License, which permits unrestricted use, distribution, and reproduction in any medium, provided the original author and source are credited.

Abstract
Current treatment for hepatocellular carcinoma consists of chemotherapy and invasive tumor removal surgery.
These forms of treatment have often been disappointing while new and natural methods to treat liver cancer may be
preferred. We tested the cytotoxic effects of the fruit mixture Galaxy ${ }^{\circledR}$ against HepG2 hepatocellular carcinoma cells
in vitro. Galaxy ${ }^{\circledR}$ was evaluated using three groups; thus, cytotoxicity bioassays were determined against: whole
product (unfractionated), the supernatant, and the particulates. Paclitaxel, a major FDA-approved chemotherapeutic
drug, was tested against HepG2 cells in order to compare the effectiveness of the Galaxy ${ }^{\circledR}$ matrices against
traditional chemotherapy. In addition, a 24 -hour bioassay was performed to compare the toxicity of Galaxy ${ }^{\circledR}$ each
hour through the incubation period. Our results have shown that unfractionated Galaxy ${ }^{\circledR}$ has significant anticancer
activity in vitro against HepG2 hepatocellular carcinoma, killing $90 \%$ of cancer cells at $26.3 \mathrm{mg} / \mathrm{mL}$. The particulates
and supernatant fractions were also cytotoxic on liver cancer. The majority of anticancer activity was present in the
particulate fraction which suggests that most of the anticancer agents are located primarily in the pulp and are
membrane bound. While Galaxy is not as effective as Paclitaxel at lower concentrations; our in vitro results clearly
show that Galaxy ${ }^{\circledR}$ (all three matrices) may be an effective product against human hepatocellular carcinoma at
higher concentrations. During the 24 -hour cycle study, unfractionated Galaxy ${ }^{\circledR}$ showed a steady linear cytotoxic
effect per hour. Collectively the data from these experiments suggest that Galaxy ${ }^{\circledR}$ merits serious consideration in
the development of future research protocols.

Keywords: Hepatocellular carcinoma; Cytotoxic effects; HepG2 cells; Galaxy

\section{Introduction}

Hepatocellular carcinoma (liver cancer; HCC) is the sixth most common cancer worldwide, resulting in 748,300 new cases diagnosed in 2008 [1]. The liver functions to absorb and store nutrients, remove toxic waste and create clotting factors that stop bleeding from injuries. When hepatocellular carcinoma is present, the body exhibits symptoms such as drastic loss of weight, swelling in the abdomen or jaundice [2]. The risk of developing liver cancer increases with factors associated with hepatitis $\mathrm{B} / \mathrm{C}$ virus, aflatoxin, iron storage disease, cirrhosis, alcoholism or obesity [3-5]. The average survival rate for hepatic cancer patients is $3-5 \%$ [6].

Treatment for liver cancer traditionally consists of surgery or chemotherapy, with often unsatisfactory results [7,8]. This disappointing data suggest the need for alternative treatments such as natural remedies. A modified diet including an increased intake of fruits and vegetables could prevent up to $20 \%$ of cancer cases and 200,000 deaths worldwide [9]. Studies of natural remedies have shown that many fruit and plant extracts contain a diverse suite of anticancer compounds. In 2010, researchers found that Camptothecin, isolated from the hairy root culture Ophiorrhiza pumila, showed anticancer activity and in 2012 that plant polyphenols contain chemopreventive properties that are cytotoxic against HCC $[10,11]$.

Galaxy ${ }^{\circledR}$ is a nutritional supplement composed of 32 bioactive ingredients. Some of the superfruits include acai, cranberry, mangosteen, and goji which are known for their antioxidant properties and have ORAC values of $425,354,251$, and $378 \mu$ moles $\mathrm{TE} / \mathrm{g}$ respectively [12]. The effects of Galaxy ${ }^{\circledR}$ have been tested on colon, liver, lung, and breast cancer cell lines in vitro and show varying degrees of cytotoxicity. Hepatic cancer is traditionally difficult to treat. Hence, the objectives of the current study were to (1) determine the in vitro cytotoxicity of Galaxy ${ }^{\circledR}$ on HepG2 cancer cells from the whole product (unfractionated), supernatant, and particulate fractions; (2) determine the cytotoxicity of Paclitaxel, an FDA-approved drug, for various cancers, against HCC; (3) investigate the cytotoxicity profile of unfractionated Galaxy ${ }^{\circledR}$ during each hour of a 24-hour incubation cycle.

\section{Materials and Methods}

\section{Cell line care}

HepG2, a perpetual cell line derived from the liver tissue of a 15year old Caucasian male, was cryogenically stored in a liquid nitrogen dewar. When needed for testing, cells were thawed in an aseptic environment and immediately placed in $7 \mathrm{~mL}$ of a growth medium which consisted of $500 \mathrm{~mL}$ of RPMI $1640,50 \mathrm{~mL}$ of fetal bovine serum, $0.15 \mathrm{~g}$ of L-glutamine and $0.75 \mathrm{~g}$ of sodium bicarbonate. Cell lines were grown in T-175 cell culture flasks at $37^{\circ}$ in an atmosphere containing $5 \% \mathrm{CO}_{2}$, and were subcultured twice a week to control the adhering cell line via trypsinization.

When the cells became confluent, trypsinization was conducted under a laminar flow hood. The T-175 flask containing HepG2 
cultures was removed from the incubator. The cap of the flask was tightened before being removed from the incubator and liberally sprayed with $70 \%$ ethanol solution before being placed in the flow hood. Growth medium, HBSS (Hank's Buffered Salt Solution) and trypsin-EDTA bottles were lightly flamed at the neck with a Bunsen Burner, sprayed with $70 \%$ ethanol, and placed in the flow hood. A waste beaker was also sanitized with ethanol and placed inside the flow hood. The liquid contents of the flask were aspirated using a GeneMate Motorized Filler/Dispenser and a $10 \mathrm{~mL}$ serological pipette tip, then discarded. Using a new $10 \mathrm{~mL}$ serological pipette tip, $25 \mathrm{~mL}$ of HBSS was used to wash the cells and then discarded. This process removed the chelating ions from the media remaining in the cell monolayer, yielding improved trypsin enzymatic activity. HBSS was introduced into and removed from the flask a second time in order to increase the productivity of this process. Cells were separated from the flask by applying an even coat of 3-5 $\mathrm{mL}$ trypsin-EDTA. After 1 minute, all but $0.5 \mathrm{~mL}$ of the trypsin-EDTA was aspirated and discarded. The cap of the T-175 flask was loosely secured to allow airflow, placed in the incubator for 10-15 minutes, and then returned to the flow hood.

The dislodged HepG2 cells were then washed off of the back wall with $10 \mathrm{~mL}$ of growth medium using a $10 \mathrm{~mL}$ serological pipette tip. The cells were then triturated 15-30 times, after which $8 \mathrm{~mL}$ of the solution were discarded. Using a $25 \mathrm{~mL}$ serological pipette tip, $23 \mathrm{~mL}$ of growth medium were then added. Finally, the flask was returned to the incubator with the cap slightly open.

\section{Dose response curves}

Dose response curves were obtained through the completion of plating, drug administration, staining, and data acquisition protocols as outlined below. Each Galaxy ${ }^{\circledR}$ dose-response curve represented three replicates per point, with $\mathrm{r}^{2}$ values ranging from 0.57 to 0.85 depending on whether it was unfractionated, supernatant, or particulate fraction.

\section{Sample preparation}

Galaxy ${ }^{\circledR}$, a nutritional supplement, was provided by Juuva. This blend contains 32 bioactive ingredients that include: "superfruits"acai, pineapple, red grape, white grape, pomegranate, red raspberry, acerola, aronia, cranberry, goji, mangosteen, elderberry, plum; "superfoods"-barley, buckwheat, flaxseed, alfalfa, wheatgrass, garlic; probiotics-Lactobacillus, Acidophilu, alpha lipoic acid; other ingrededients - cayenne pepper, green tea leaf extract, soybean, coenzyme Q10, magnesium steaiate, ascorbic acid, citric acid, xanthan gum, DMAE. Bioassay data from this product were collected using the fractionated and unfractionated samples. After thorough agitation of the Galaxy ${ }^{\circledR}$ container, in order to provide uniform distribution of its contents, $10 \mathrm{~mL}$ of sample was aspirated using the GeneMate Motorized Pipet Filler/Dispenser and a $10 \mathrm{~mL}$ serological pipette tip and expelled into a $100 \mathrm{~mL}$ Erlenmeyer Flask or $15 \mathrm{~mL}$ conical tube. Further details regarding the preparation of unfractionated product, supernatant, and particulate fraction are detailed below. Unfractionated product: A $100 \mathrm{~mL}$ Erlenmeyer Flask was filled with $10 \mathrm{~mL}$ of Galaxy ${ }^{\circledR}$ solution. Subsequently, a magnetic stir bar was placed inside the flask of solution. This flask was positioned on top of a Cimarec hot plate, and set at $40^{\circ} \mathrm{C}$ on a low spinning frequency in order to avoid splashing. Compressed air was blown into the flask to minimize drying time while avoiding splashing. The Erlenmeyer Flask was left for 24 hours to allow for complete dehydration. The dehydrated sample was weighed on an Ohaus precision standard digital scale, resuspended with distilled water to the original $10 \mathrm{~mL}$ volume and left for 24 hours using a stirring bar. Supernatant: A $15 \mathrm{~mL}$ conical tube was filled with $10 \mathrm{~mL}$ of Galaxy ${ }^{\circledR}$ sample, and then centrifuged for 8 minutes. The supernatant was then transferred into a $100 \mathrm{~mL}$ Erlenmeyer Flask. The sample was again placed in the centrifuge and spun for an additional 8 minutes. The remaining supernatant was transferred into the $100 \mathrm{~mL}$ Erlenmeyer Flask and weighed. The sample was set on the hot plate at a low spin frequency and dehydrated for 24 hours with the aid of the compressed air. After 24 hours, the dehydrated sample was weighed, resuspended with distilled water to the original $10 \mathrm{~mL}$ volume, and left for 24 hours using a stirring bar set on the hot plate at a low spin frequency. Particulate fraction: A $15 \mathrm{~mL}$ conical tube was filled with $10 \mathrm{~mL}$ of Galaxy ${ }^{\circledR}$ sample, and then centrifuged for 8 minutes. The supernatant was then discarded. After repeating the 8 minute cycle and discarding the remaining supernatant, the wet particulate fraction sample was weighed. The sample was then left for 24 hours for complete dehydration with the aid of an air tube. After 24 hours, the dehydrated sample was weighed, resuspended with distilled water to the original $10 \mathrm{~mL}$ volume and left for 24 hours using a stirring bar set on the hot plate at a low spin frequency.

\section{Plating}

The plating process was initiated by performing the protocol outlined in the cell line care section of this article with one exception: rather than discarding a fraction of the cells immediately after passaging, a $0.5 \mathrm{~mL}$ sample of cell solution was withdrawn, leaving the remainder. The $0.5 \mathrm{~mL}$ sample was transferred to an Eppendorf Tube, and taken out of the flow hood. This sample was assessed using trypan blue dye. On a strip of parafilm, $20 \mu \mathrm{L}$ of cell solution was mixed with $20 \mu \mathrm{L}$ trypan blue by triturating thoroughly. After which, $10 \mu \mathrm{L}$ of the resultant mixture was transferred onto a hemocytometer and viewed under a microscope. The live cells absorbed the dye and immediately pumped it out of their membranes becoming a translucent color, while the dead cells absorbed the dye becoming blue in color. The live cells were counted in the four cornered sections of the hemocytometer and divided by four to find an average number of cells per the $1 / 10,000 \mathrm{~cm}^{3}$ cornered section. The average was multiplied by 20,000 to account for the trypan blue dilution and to find the total number of cells in $1 \mathrm{~mL}$ of cell solution. To achieve 1 million cells per milliliter, 1 million was divided by the cell count. This amount was then extracted from the $15 \mathrm{~mL}$ conical tube in the flow hood and transferred into a pipettor basin using a $1 \mathrm{~mL}$ serological pipette tip. A total of $15 \mathrm{~mL}$ of growth media was then added to the pipettor basin and triturated $15-30$ times using a $10 \mathrm{~mL}$ serological pipette tip. Using a multipipettor, $150 \mu \mathrm{L}$ of cell solution were then added to each well of a 96-well flat-bottom plate. This step was repeated for two additional plates. The plates were then taken out of the flow hood, labeled, and transferred into the incubator and left for 24 hours.

\section{Sample administration}

Twelve separate dosages of a Galaxy ${ }^{\circledR}$ sample were investigated during each iteration of the dose response assay. Dosages were prepared by mixing between 4 and $200 \mu \mathrm{L}$ of the product from the drug preparation protocol with a sufficient amount of media to yield a final volume of $0.6 \mathrm{~mL}$. This produced 12 separate $0.6 \mathrm{~mL}$ volumes of fluid, with linearly increasing sample concentrations. Due to differences in the activity between unfractionated, supernatant, and 
particulate fractions, the dose preparation range maintained a large degree of variation. The resultant mixtures were vortexed for 30 seconds immediately prior to administration. Within the flow hood, each individual concentration of drug was delivered into 3 adjacent wells of the 96-well plate identified in the plating procedure above. The location of each administered dose was randomized, with the exception of the 9 control wells. Negative control wells were located at B2-4, C5-7, and F8-10. Control wells received an additional $50 \mu \mathrm{L}$ of media, while each treated well received $50 \mu \mathrm{L}$ of its respective product mixture. Once each plate of cells was administered with the requisite quantity of Galaxy ${ }^{\circledR}$ substrate, the plates were returned to the incubator and allowed to culture for an additional 24 hours.

\section{Staining and data acquisition}

Following incubation with drug for 24 hours, plates were withdrawn from the incubator and percent viability of the HepG2 cells was determined via the SRB assay [13]. After the stained plates were dry, each well was filled with $75 \mu \mathrm{L}$ of $1 \mathrm{mM}$ Tris base. Plates were agitated and placed into a BioTek Gen 5 Microplate Reader. Absorption of the sulforhodamine B taken up by cells surviving treatment was measured at a wavelength of $570 \mathrm{~nm}$. After all data were obtained, the absorbances of the various drugged wells were compared to the average absorbance of the control wells to obtain a percent viability vs dose response curve.

\section{Timed response}

In order to obtain a 24 hour response curve, flat bottomed 96-well plates were cultured with HepG2 cells in accordance with the plating protocol detailed above. After 24 hours, each plate was administered doses of Galaxy ${ }^{\circledR}$ unfractionated product such that the final product to volume concentration of the drugged well was either $5,10,15$, or 20 $\mathrm{mg} / \mathrm{mL}$. Each plate was also administered a $0.02 \mathrm{mg} / \mathrm{mL}$ and a 0.0005 $\mathrm{mg} / \mathrm{mL}$ dose of Paclitaxel. Each dose was tested in at least 12 wells. One hour after completion of plate drugging, the first plate was removed from the incubator and stained according to the SRB assay. Two hours after plate drugging was completed, the second plate was removed and likewise stained. This process continued in hourly intervals until the dose response data was completed for hours 1 to 24 subsequent to treating HepG2 cultures with unfractionated product. Plates were read according to the procedures above, and results were compared with cell viability followed as a function of time.

\section{Results}

\section{Cytotoxicity}

Historically, the cytotoxic effects of Galaxy ${ }^{\circledR}$ have varied significantly between different types of cancer. In vitro data shows that the product's effects on MDA-MB-231 human breast cancer cells are quite potent with an $\mathrm{EC}_{50}$ of $2.3 \mathrm{mg} / \mathrm{mL}$ [12]. However, when the unfractionated Galaxy ${ }^{\circledR}$ was tested against HepG2 HCC, the cells were more difficult to kill. Effects of Galaxy ${ }^{\circledR}$ were tested in vitro with varying dosages. To test the HCC cytotoxicity of Galaxy ${ }^{\circledR}, 15$ bioassays were completed for the unfractionated product as well as 15 bioassays each from the supernatant and particulate fractions. The dose response curve suggests a divergence of HEPG2 responses to Galaxy ${ }^{\circledR}$. When treated with the unfractionated Galaxy ${ }^{\circledR}$, HepG2 cell mortality vs product concentration demonstrated a linear relationship from 0 to $30 \mathrm{mg} / \mathrm{mL}$. Higher concentrations, however, did not show increased anticancer activity. Simple linear regression showed a predicted $\mathrm{EC}_{50}$ of $20.2 \mathrm{mg} / \mathrm{mL}$. Approaching $30 \mathrm{mg} / \mathrm{mL}$, unfractionated Galaxy $^{\circledR}$ demonstrated up to $80 \%$ cytotoxicity to HepG2 liver cancer (Figure 1). When fractionated into the supernatant, the dose response curve changed and demonstrated a decrease in cytotoxic activity. The same linear relationship exists, however, with the $\mathrm{EC}_{50}$ value increasing to $39.0 \mathrm{mg} / \mathrm{mL}$ (Figure 2). Particulate fractions demonstrate the greatest potency on HepG2 liver cancer cells. The $\mathrm{EC}_{50}$ value drops to $7.02 \mathrm{mg} / \mathrm{mL}$ while obtaining roughly $80 \%$ mortality of liver cancer cells at $11 \mathrm{mg} / \mathrm{mL}$ (Figure 3 )

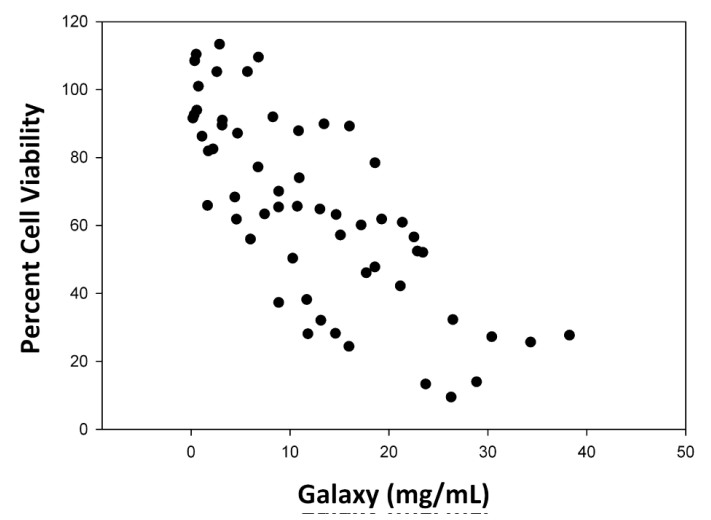

Figure 1: Effect of unfractionated Galaxy ${ }^{\circledR}$ product against HepG2 human liver cancer cells.

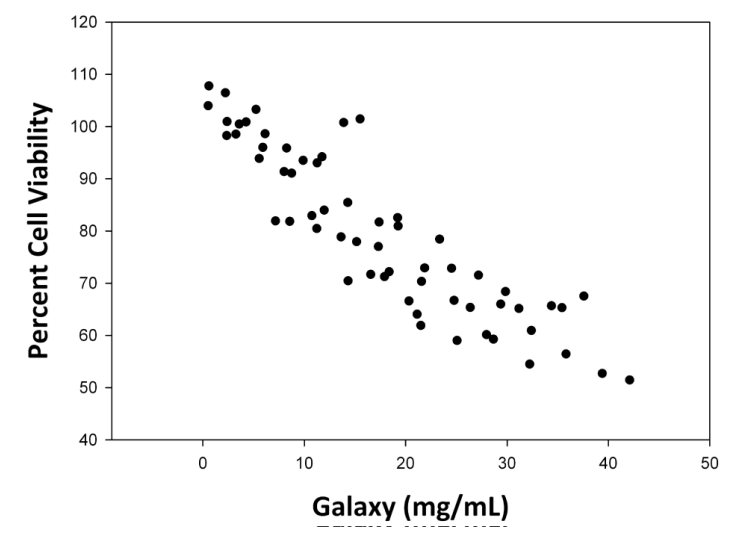

Figure 2: Effect of Galaxy ${ }^{\circledR}$ supernatant against HepG2 human liver cancer cells.

\section{Galaxy ${ }^{\circledR}$ compared to paclitaxel}

In order to compare the efficacy of Galaxy ${ }^{\circledR}$ as an anticancer product, three in vitro bioassays were completed with Paclitaxel against HepG2 hepatic cancer cells [14]. A linear relationship similar to Galaxy ${ }^{\mathbb{B}}$ results when the percent viability is graphed against $\mathrm{mg} / \mathrm{mL}$ of a diluted Paclitaxel serum. Paclitaxel shows an $\mathrm{EC}_{50}$ of 0.02 $\mathrm{mg} / \mathrm{mL}$ and an average of $92 \%$ cell death at $0.04 \mathrm{mg} / \mathrm{mL}$ (Figure 4). 


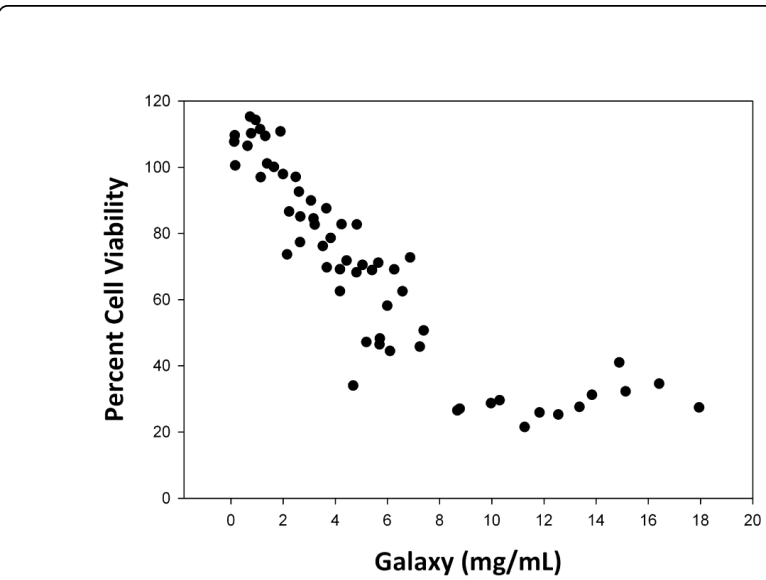

Figure 3: Effect of Galaxy ${ }^{\circledR}$ particulates against HepG2 human liver cancer cells.

The $\mathrm{EC}_{50}$ of Paclitaxel is $350 \mathrm{x}$ less and therefore more cytotoxic than the particulate fraction of Galaxy ${ }^{\circledR}$. The highest value of cell mortality with the particulate fraction was $79 \%$ at $11.3 \mathrm{mg} / \mathrm{mL}$. Paclitaxel kills 14\% more cancer cells at a concentration 283x less than the particulate fraction of Galaxy ${ }^{\circledR}$. However, in an ongoing cooperative study between Brigham Young University and China Pharmaceutical University, the data show that Galaxy ${ }^{\circledR}$ can shrink liver tumors in mice while also boosting the immune system. Conversely, Paclitaxel shrinks liver tumors but depresses the immune system significantly [15].

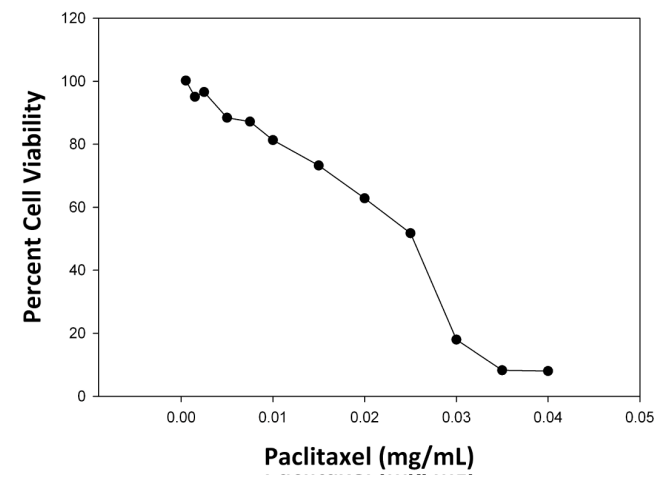

Figure 4: Effect of Paclitaxel against HepG2 human liver cancer cells.

\section{Hour profile}

To determine the hourly toxicity of Galaxy ${ }^{\circledR}$, a 24 hour bioassay (samples taken every hour) was performed. Cells were incubated in 96-well plates for 24 hours and then administered varying dosages of unfractionated Galaxy ${ }^{\circledR}$ product. Surviving cells were then arrested and dyed every hour. The hourly profile did not indicate a specific hour of toxic activity, but rather the data clearly indicate a steady linear cytotoxic effect per hour (Figure 5). Dosages ranging from 5 $\mathrm{mg} / \mathrm{mL}$ to $20 \mathrm{mg} / \mathrm{mL}$ did not result in significantly different values of cell viability. All four dosages approached $50 \%$ cell viability after the 24 hour period and caused significant mortality linear through time.

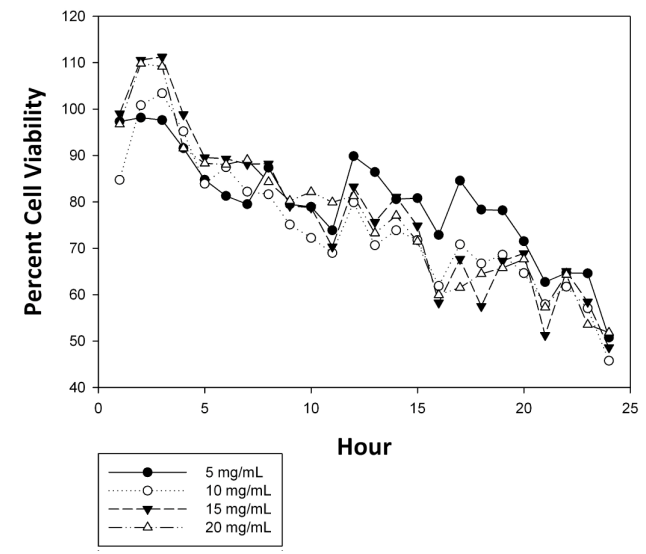

Figure 5: 24 hour toxicity profile of unfractionated Galaxy ${ }^{\circledR}$ product against HepG2 human liver cancer cells.

\section{Discussion}

Cancer treatment is making significant progress by decreasing deaths and increasing life expectancy of patients. However, in contrast to most cancer types, liver cancer is on the rise in the United States and in many regions of the world [16]. Liver and intrahepatic bile duct cancer has risen to an estimated 33,190 new cases in 2014, and an estimated 23,000 deaths. Of patients with hepatic cancer, a low rate of $16.6 \%$ survive 5 years or longer [17]. Today, many different methods to treat this disease have found success and failure. Traditional chemotherapy treatment has proven to be relatively ineffective against HCC and effectiveness often decreases with each treatment due to increased resistance of the hepatic cancer cells [18]. In light of the problems with traditional chemotherapeutic treatments, many patients have found success with herbal remedies that include consuming a diverse group of natural fruits and vegetables. This approach will continue to add new insights into novel compounds and mixtures that will add to our improved chemotherapy drug arsenal.

Our current data on Galaxy ${ }^{\circledR}$ have shown significant anticancer activity in vitro against HepG2 hepatocellular carcinoma. This blend of fruits has demonstrated a mortality rate of up to $90 \%$ on cancer cells at $26.3 \mathrm{mg} / \mathrm{mL}$ of unfractionated product. When fractionated into supernatant and particulate components, the $\mathrm{EC}_{50}$ indicates that the anticancer compounds are less abundant in the non-pulp containing juice of the fruits and more concentrated within the particulates. The particulate $\mathrm{EC}_{50}$ value was $7.02 \mathrm{mg} / \mathrm{mL}$ compared to the supernatant which was $39.0 \mathrm{mg} / \mathrm{mL}$. Hence, the particulate fraction is about $5.5 \mathrm{x}$ more effective than the supernatant and almost $3 \mathrm{x}$ as effective as the unfractionated Galaxy ${ }^{\circledR}$. The particulate toxicity to HCC is similar to previous trends observed with the freeze dried product. However, freeze dried Galaxy ${ }^{\circledR}$ appears to be more toxic than the particulate fraction [12]. The data suggest that the most effective anticancer agents present in Galaxy ${ }^{\circledR}$ are contained in the pulp and are membrane bound. 


\begin{tabular}{|l|l|l|}
\hline & $\begin{array}{l}\mathrm{EC}_{50} \\
(\mathrm{mg} / \mathrm{mL})\end{array}$ & $\begin{array}{l}\text { Cell death } \\
(\text { at 10 } \mathrm{mg} / \mathrm{mL})\end{array}$ \\
\hline Whole Product & 20.2 & $30 \%$ \\
\hline Supernatant & 39.0 & $11 \%$ \\
\hline Particulate & 7.0 & $74 \%$ \\
\hline Paclitaxel & 0.02 & $70 \%^{*}$ \\
\hline
\end{tabular}

Table 1: Summary of the $\mathrm{EC}_{50}$ and percent cell death for whole product, supernatant, particulate, and Paclitaxel. ${ }^{*}$ Cell death of Paclitaxel was measured at $0.03 \mathrm{mg} / \mathrm{mL}$.

Polyphenolic compounds in fruit have been linked to anticancer activity and normally reside in the skin and pulp of fruit [19]. Research has shown that extracted phenolic compounds have about $50 \%$ more cytotoxic activity on HepG2 cancer cells than crude extracts of the same fruit [20]. Galaxy ${ }^{\circledR}$ particulate data is encouraging of such phenolic activity; however, further investigation of the phenolic content of Galaxy ${ }^{\circledR}$ is needed to confirm such a hypothesis. The data for the two fractions demonstrate a close-fit negative linear regression of concentration of cell viability Vs concentration. However, the unfractionated product exhibits a wider range of data variability with a looser fitting linear regression. Linear regression analysis of the crude product shows an $\mathrm{r}^{2}$ value of 0.57 compared to 0.81 and 0.85 for the supernatant and particulate fractions respectively. This supports the hypothesis that the main anticancer compounds are located within the particulates of the fruit blend because when the drug was unfractionated, there was no regulation of the amount of particulates vs supernatant per bioassay administration. Despite the potent effects of the Galaxy ${ }^{\circledR}$ pellet on HepG2 cancer cells, Paclitaxel still proved to be $350 \mathrm{x}$ more effective in vitro with an $\mathrm{EC}_{50}$ of 0.02 $\mathrm{mg} / \mathrm{mL}$. This finding is interesting due to the often surprisingly ineffective nature of chemotherapy use in vivo against HCC [14].

Many studies have isolated and tested active ingredients within different natural products that have cytotoxic effects on cancer cells. It has been discovered that cranberry, lemon, apple, strawberry, and red grape have strong cytotoxic effects on HepG2 cancer cells with an $\mathrm{EC}_{50}$ of $14.5,30.6,49.4,56.3$ and $71.0 \mathrm{mg} / \mathrm{mL}$ respectively [21]. However, in comparison to many studies of isolated herbal-based compounds and whole herb concoctions, the isolated compounds show less anticancer activity [22]. In another study, the antioxidant effects of apples, blueberries, grapes, and oranges, as well as a mixture of all four were observed. The $\mathrm{EC}_{50}$ of each fruit was $5 \mathrm{x}$ higher than that of the combination of the fruits. It is hypothesized that the different phytochemical profiles of the fruits and the synergistic activity of combinations of different polyphenolic compounds may lead to increased anticancer activity when compared to the single compounds [23]. Thus, it is not surprising to see that Galaxy ${ }^{\circledR}$, as a mixture of many different compounds, shows more cytotoxicity than many single components that have been tested against HepG2 cancer cells.

Galaxy ${ }^{\circledR}$ fruit juice is not marketed as a medicinal drug; however, as a nutritional supplement this product has a blend of fruits and vegetables that is lethal to hepatic cancer cells in vitro. More research is needed to confirm which compounds within the blend are the most cytotoxic and by which mechanism they synergistically induce cancer cell death. In addition, it is still unknown whether Galaxy ${ }^{\circledR}$ kills by apoptosis or necrosis.

\section{Conclusion}

Galaxy ${ }^{\circledR}$ has clear in vitro anticancer properties against HepG2 cancer cells. Specifically, the particulate fraction shows strong toxicity against liver cancer cells with an $\mathrm{EC}_{50}$ of $7.02 \mathrm{mg} / \mathrm{mL}$ which is $3 \mathrm{x}$ more effective than unfractionated Galaxy ${ }^{\circledR}$. This suggests that the most effective anticancer agents of the Galaxy ${ }^{\circledR}$ fruits are membrane bound in the pulp. The effect of Galaxy ${ }^{\circledR}$ on HepG2 cancer cells over a 24 hour period was studied and it was found that there is no specific time period in which toxic activity takes place, but rather there is a linear decrease in cell viability each hour. In vitro, Galaxy ${ }^{\circledR}$ is not as effective as a common anticancer drug Paclitaxel, which had an $\mathrm{EC}_{50}$ of $0.02 \mathrm{mg} / \mathrm{mL}$. However, the cytotoxicity profiles of Galaxy ${ }^{\circledR}$ (i.e., the graphic profiles) have similar plots. It simply kills the HCC at higher concentrations. Additional investigations must be designed to discover the specific anticancer compounds within Galaxy ${ }^{\circledR}$ and the mechanism by which these compounds synergistically induce cancer cell death. Preliminary data from a current joint project with Brigham Young University and China Pharmaceutical University show that Galaxy ${ }^{\circledR}$ can shrink liver tumors in mice while also boosting the immune system [15]. Paclitaxel also shrinks liver tumors in mice, but it clearly depresses the immune system. These experiments are ongoing.

\section{References}

1. Jemal A, Bray F, Center MM, Ferlay J, Ward E, et al. (2011) Global cancer statistics. CA Cancer J Clin 61: 69-90.

2. al-Sarraf M, Go TS, Kithier K, Vaitkevicius VK (1974) Proceedings: Primary liver cancer. A review of the clinical features, blood groups, serum enzymes, therapy, and survival of 65 cases. Cancer 33: 574-582.

3. Bosch FX, Ribes J, Borràs J (1999) Epidemiology of primary liver cancer. Semin Liver Dis 19: 271-285.

4. Pang R, Tse E, Poon RT (2006) Molecular pathways in hepatocellular carcinoma. Cancer Lett 240: 157-169.

5. Neuschwander-Tetri BA, Caldwell SH (2003) Nonalcoholic steatohepatitis: summary of an AASLD Single Topic Conference. Hepatology 37: 1202-1219.

6. Parkin DM, Pisani P, Ferlay J (1999) Global cancer statistics. CA Cancer J Clin 49: 33-64, 1.

7. Takayama T, Makuuchi M, Hirohashi S, Sakamoto M, Yamamoto J, et al. (1998) Early hepatocellular carcinoma as an entity with a high rate of surgical cure. Hepatology 28: 1241-1246.

8. Horgan AM, Dawson LA, Swaminath A, Knox JJ (2012) Sorafenib and radiation therapy for the treatment of advanced hepatocellular carcinoma. J Gastrointest Cancer 43: 344-348.

9. Amin AR, Kucuk O, Khuri FR, Shin DM (2009) Perspectives for cancer prevention with natural compounds. J Clin Oncol 27: 2712-2725.

10. M Yamazaki, T Asano, Y Yamazaki, S Sirikantaramas, H Sudo, et al. (2010) Biosynthetic system of camptothecin: An anticancer plant product. Pure and Applied Chemistry 82: 213-218.

11. Stagos D, Amoutzias GD, Matakos A, Spyrou A, Tsatsakis AM, et al. (2012) Chemoprevention of liver cancer by plant polyphenols. Food Chem Toxicol 50: 2155-2170.

12. Booth GM, Parker TL, Lee CM (2012) Antioxidant, anticancer activity, and other health effects of a nutritional supplement $\left(G a l a x y^{\circ}\right)$. Structure and Function of Food Engineering

13. Skehan P, Storeng R, Scudiero D, Monks A, McMahon J, et al. (1990) New colorimetric cytotoxicity assay for anticancer-drug screening. J Natl Cancer Inst 82: 1107-1112.

14. Ho CK, Huang YL, Chen CC (2002) Garcinone E, a xanthone derivative, has potent cytotoxic effect against hepatocellular carcinoma cell lines. Planta Med 68: 975-979. 
Citation: Jeffs DB, Martin AC, Rosenvall ER, Fillmore T, Barrett KE, et al. (2014) Cytotoxicity of Nutritional Supplement Galaxy® against HepG2 Hepatocellular Carcinoma . Altern Integ Med 3: 175. doi:10.4172/2327-5162.1000175

Page 6 of 6

15. Yang, Yong (2014) Personal communication

16. Jemal A, Ward E, Thun M (2010) Declining death rates reflect progress against cancer. PLoS One 5: e9584.

17. Surveillance Research Program, National Cancer Institute Fast stats: an interactive tool for access to SEER cancer statistics November 10, 2011.

18. Huang CC, Wu MC, Xu GW, Li DZ, Cheng H, et al. (1992) Overexpression of the MDR1 gene and P-glycoprotein in human hepatocellular carcinoma. J Natl Cancer Inst 84: 262-264.

19. Lee JH, Talcott ST (2004) Fruit maturity and juice extraction influences ellagic acid derivatives and other antioxidant polyphenolics in muscadine grapes. J Agric Food Chem 52: 361-366.

20. Yi W, Akoh CC, Fischer J, Krewer G (2006) Effects of phenolic compounds in blueberries and muscadine grapes on HepG2 cell viability and apoptosis. Food Research International 39: 628-638.
21. Sun J, Chu YF, Wu Xianzhong, Hai Liu R (2002) Antioxidant and antiproliferative activities of common fruits. Journal of Agricultural and Food Chemistry 50: 449-54.

22. Machana S, Weerapreeyakul N, Barusrux S, Thumanu K, Tanthanuch W (2012) Synergistic anticancer effect of the extracts from Polyalthia evecta caused apoptosis in human hepatoma (HepG2) Cells. Asian Pacific Journal of Tropical Biomedicine 2: 589-596.

23. Liu RH (2004) Potential synergy of phytochemicals in cancer prevention: mechanism of action. J Nutr 134: 3479S-3485S. 